Pacific Journal of Mathematics

COMPLETIONS AND CLASSICAL LOCALIZATIONS OF

GERHARD O. MichleR 


\section{COMPLETIONS AND CLASSICAL LOCALIZATIONS OF RIGHT NOETHERIAN RINGS}

\section{J. LAMBEK AND G. MICHLER}

Given a right Noetherian ring $R$ and a prime ideal $P$ of $R$, the injective hull of the right $R$-module $R / P$ is a finite power of a uniquely determined indecomposable injective $I_{P}$. One forms the ring of right quotients $R_{P}$ of $R$ relative to $I_{P}$ and the right ideal $M=P R_{P}$ of $R_{P}$ generated by $P$. The $M$-adic and $I_{P}$-adic topologies are compared; they turn out to coincide on every finitely generated $R_{P}$-module when $R_{P}$ is a classical quasi-local ring with maximal ideal $M$. This condition also implies that $R$ satisfies the right Ore condition with respect to the multiplicative set $\mathscr{C}(P)$ introduced by Goldie, that the $M$-adic completion $\hat{R}_{P}$ of $R_{P}$ is the bicommutator of $I_{P}$, and that $\hat{R}_{P}$ is an $n$ by $n$ matrix ring over a complete local ring.

Introduction. If $P$ is a prime ideal of the commutative Noetherian ring $R$, then, by a theorem of Matlis [8], the completion $\hat{R}_{P}$ of the ring of quotients of $R$ at $P$ is the bicommutator of the injective hull of the $R$-module $R / P$. Recently Kuzmanovich [5] proved an analogous result for Noetherian Dedekind prime rings. Both these results are special cases of Theorem 6 below: Let $P$ be a two-sided prime ideal of the right Noetherian ring $R$, and assume that the ring of right quotients $R_{P}$ at $P$ is a classical quasi-local ring with maximal ideal $M=P R_{P}$, that is, $R_{P} / M$ is a simple Artinian ring and, for every right ideal $E$ of $R_{P}, \bigcap_{n=1}^{\infty} E+M^{n}=E$. Then the bicommutator of the $R$-injective hull of $R / P$ is the $M$-adic completion of $R_{P}$. The hypothesis of Theorem 6 is satisfied by the prime ideals of the enveloping algebra of a finitely generated nilpotent Lie algebra, by the augmentation ideal of a group ring of a finite group over a right Noetherian prime ring of characteristic zero, and by the nonidempotent prime ideals of a right and left Noetherian hereditary prime ring.

These results are consequences of Theorem 5 , which states that $R_{P}$ is a classical quasi-local ring with maximal ideal $M$ if and only if $R_{P} / M$ is a simple Artinian ring, and, on any finitely generated $R_{P^{-}}$ module, the $M$-adic topology coincides with the $I_{P}$-adic topology. Here $I_{P}$ denotes the unique (up to isomorphism) $P$-torsionfree indecomposable injective $R$-module with associated prime $P$. By [7], Theorem 3.9, the injective hull $I_{R}(R / P)$ of the $R$-module $R / P$ is isomorphic to a direct sum of $g$ copies of $I_{P}$, where $g$ is the Goldie dimension of the prime ring $R / P$. Thus the bicommutators of $I_{P}$ and $I_{R}(R / P)$ are isomorphic. 
Concerning terminology, we refer to [6], [7], and [8]. All rings are associative and have a unity element. Modules are right $R$-modules and unitary. We put

$$
\mathscr{C}(P)=\left\{c \in R \mid \forall_{r \in R} c r \in P \Longrightarrow r \in P\right\} .
$$

We begin by comparing topologies, generalizing the known result when $R$ is commutative [6].

Proposition 1. If $R$ satisfies the right Ore condition with respect to $\mathscr{C}(P)$, then on any finitely generated $R_{P}$-module the $I_{P}$-adic topology contains the $M$-adic topology, where $M=P R_{P}$.

Proof. Let $G$ be any finitely generated $R_{P}$-module. Take any fundamental open neighborhood $G M^{n}$ of zero in the $M$-adic topology. We claim that $G M^{n}$ is also open in the $I_{P}$-adic topology, in fact, $G / G M^{n} \in \mathscr{F}$, the class of all $R_{P}$-modules isomorphic to submodules of finite powers of $I_{P}$.

Since $\mathscr{F}$ is closed under module extensions, and since

$$
G M^{n} \sqsubseteq G M^{n-1} \sqsubseteq \cdots \subseteq G M \subseteq G,
$$

it suffices to show that $G M^{k} / G M^{k-1} \in \mathscr{F}$. Put $H=G M^{k}$, then $H$ is a finitely generated $R_{P}$-module. Now $R_{P} / M$ is a simple Artinian ring, by [7], Theorem 5.6. Hence $H / H M$ is isomorphic to a finite direct sum of minimal right ideals of $R_{P} / M$.

It remains to show that $R_{P} / M \in \mathscr{F}$. Indeed, in view of [7], Lemma 5.4, the mapping $R \stackrel{h}{\longrightarrow} R_{P} \rightarrow R_{P} / M$ has kernel $P$, and so $R_{P} / M$ may be regarded as an $R$-module extension of $R / P$. Actually, it is an essential extension; for, if $0 \neq[q] \in R_{P} / M$, then $q \notin M$, but $q c \in h(R)$, for some $c \in \mathscr{C}(P)$, and $g c \notin h(P)$, since otherwise $q=q c c^{-1} \in$ $h(P) R_{P}=P R_{P}=M$. Thus $R_{P} / M$ is isomorphic to an $R$-submodule of $I_{R}(R / P)=I_{P}^{g}$. By [7], Theorem 5.6, $R_{P} / M$ is torsionfree and divisible, hence $R_{P} / M$ is also isomorphic to an $R_{P}$-submodule of $I_{P}^{g}$, and so $R_{P} / M \in \mathscr{F}$.

This completes the proof. In the converse direction we have the following result. We remark that condition (1) plays an important rôle in [4], Theorem 5.3.

Proposition 2. Suppose $M=P R_{P}$ is a two-sided ideal of $R_{P}$. Then $(1) \Rightarrow(2) \Rightarrow(3)$ :

(1) For each right ideal $E$ of $R_{P}$ there exists a natural number $n$ such that $E \cap M^{n} \subseteq E M$.

(2) For each element $i \in I_{P}$ there exists a natural number $n$ such that $i M^{n}=0$. 
(3) On any finitely generated $R_{P}$-module the $I_{P}$-adic topology is contained in the M-adic topology.

Proof. Assume (1). Let $0 \neq i \in I_{P}$, and put $F=\left\{q \in R_{P} \mid i q=0\right\}$, $E=\left\{q \in R_{P} \mid q M \leqq F\right\}$. Note that $E M \leqq F \leqq E$. Pick $n$ so that $E \cap$ $M^{n} \subseteq E M$, then $E \cap\left(M^{n}+F\right)=\left(E \cap M^{n}\right)+F=F$. Since $I_{P}$ is indecomposable, $F$ is meet-irreducible, hence $F=E$ or $M^{n}+F=F$. We shall prove that $F \neq E$, hence $M^{n} \subseteq F$, and so (1) implies (2).

As $P$ is the associated prime ideal of the $R$-module $I_{P}, P$ is the right annihilator of some nonzero $R$-submodule $U$ of $I_{P}$. Putting $V=$ $U R_{P}$, we see that $V M=0$ and $V \neq 0$. Now $i R_{P} \cong R_{P} / F$, hence $0 \neq$ $V \cap i R_{P} \cong G / F$, say, where $F \cong G \leqq E, F \neq G$, hence $F \neq E$, as remained to be shown.

Assume (2), and let $G$ be a finitely generated $R_{P}$-module. Take any fundamental open neighborhood of zero in the $I_{P}$-adic topology. By definition, this has the form $\operatorname{Ker} f$, where $f: G \rightarrow I_{p}^{n}$ for some positive integer $n$. Let $p_{k}: I_{p}^{n} \rightarrow I_{P}$ be the canonical projections, with $k=1,2, \cdots, n$, and put $G_{k}=p_{k}(f(G))$. Then $G_{k}$ is a finitely generated $R_{P}$-submodule of $I_{P}$.

By assumption, there is a natural number $u(k)$ such that $G_{k} M^{u(k)}=$ 0 . Let $u=\operatorname{Max}\{u(1), \cdots, u(n)\}$, then $f(G) M^{u}=0$, hence Ker $f$ contains $G M^{u}$, a fundamental open neighborhood of 0 in the $M$-adic topology. It follows that every open set in the $I_{P}$-adic topology is also open in the $M$-adic topology. Thus $(2) \Rightarrow(3)$, and the proof is complete.

We know from [7], Lemma 5.2, that for each element $q \in R_{P}$ there exists an element $c \in \mathscr{C}(P)$ such that $q c \in h(R)$, where $h: R \rightarrow R_{P}$ canonically. This does not imply that $R_{P}$ is the classical ring of quotients of $h(R)$ with denominators in $h(\mathscr{C}(P))$, unless $R$ satisfies the right Ore condition with respect to $\mathscr{C}(P)$. (See [7], Proposition 5.5.) However, we have the following.

Proposition 3. Let $P$ be a two-sided prime ideal of the right Noetherian ring $R$, and assume that $M=P R_{P}$ is a maximal two-sided ideal of $R_{P}$ such that $R_{P} / M$ is Artinian. Then, for every integer $n \geqq 1$, $R_{P} / M^{n}$ is the classical ring of right quotients of $h(R) /\left(M^{n} \cap h(R)\right)$, and its elements have the form $[h(r)][h(c)]^{-1}$, with $r \in R$ and $c \in \mathscr{C}(P)$.

We could deduce this from [9], Theorem 2.4, by first proving that the ideals $h^{-1}\left(M^{n}\right)$ are the $n$th symbolic powers $P^{(n)}$ defined there in a different fashion. However, it is a bit quicker to deduce this directly from the following result by Small. (See [10], Theorem 1.)

Suppose $P$ is the prime radical of the right Noetherian ring $S$, and $\mathscr{C}$ is a multiplicatively closed subset of $S$ consisting of elements with zero right annihilators. Suppose the classical ring of right quotients of $S / P$ has elements of the form $[s][c]^{-1}$, with $s \in S$ and $c \epsilon$ 
$\mathscr{C}$. Then $S$ satisfies the right Ore condition with respect to $\mathscr{C}$ and has a classical ring of right quotients with elements of the form $s c^{-1}$.

Proof. In [7], Theorem 5.6, in the proof of the implication $(1) \Rightarrow$ (2), it is shown that $R_{P} / M$ is the classical ring of right quotients of $R / P$, and that its elements have the form $[r][c]^{-1}$, where $r \in R$, and $c \in \mathscr{C}(P)$. Since $h^{-1}(M)=P$, by [7], Lemma 5.4, the result holds for $n=1$.

To obtain the result for $n=2$, we shall apply Small's Theorem to the ring $S=h(R) /\left(M^{2} \cap h(R)\right)$. To this purpose we must show that the elements of $h(\mathscr{C}(P))$ modulo $M^{2}$ have zero right annihilators. In fact, we shall see that they have left inverse in $R_{P} / M^{2}$.

Take any $c \in \mathscr{C}(P)$. In view of the case $n=1$, we have $R_{P}=$ $R_{P} c+M$. Hence $M=M R_{P}=M c+M^{2}$, and so $R_{P}=R_{P} c+M c+M^{2}=$ $R_{P} c+^{\cdot} M^{2}$. By Small's Theorem, $R_{P} / M^{2}$ is the classical ring of right quotients of $h(R) /\left(M^{2} \cap h(R)\right)$, and denominators may be taken in $\mathscr{C}(P)$ modulo $M^{2}$.

Repeating the same argument, we see that $R_{P}=R_{P} c+M^{3}$, and that $R_{P} / M^{3}$ is the classical ring of right quotients of $h(R) /\left(M^{3} \cap h(R)\right)$, with denominators in $\mathscr{C}(P)$ modulo $M^{3}$. Etc, etc.

In accordance with [7], we call the ring $S$ a classical quasi-local ring if it is right Noetherian, it has a maximal ideal $M$ such that $S / M$ is Artinian, and every right ideal of $S$ is closed in the $M$-adic topology. In view of the following lemma, this implies that $M$ is the Jacobson radical of $S$.

Lemma 4. Suppose $M$ is a primitive ideal of the ring $S$, and every finitely generated right ideal of $S$ is closed in the $M$-adic topology. Then $M$ is the Jacobson radical of $S$.

Proof. The first assumption assures that $M$ contains the radical. We claim the second assumption implies the converse. We shall prove that if $E$ is any right ideal of $S$ and $M+E=S$ then $E=S$.

Suppose $M+E=S$. Without loss in generality, we may take $E$ to be finitely generated. Now $M=S M=M^{2}+E M$, hence $M^{2}+E=$ $M^{2}+E M+E=M+E=S$. Similarly $M^{3}+E=S$, and so on. Hence the $M$-adic closure $\bigcap_{n=1}^{\infty}\left(E+M^{n}\right)$ of $E$ is also $S$. By assumption, $E$ is closed, hence $E=S$.

THEOREM 5. Let $P$ be a two-sided prime ideal of the right Noetherian ring $R$, and put $M=P R_{P}$, where $R_{P}$ is the ring of right quotients of $R$ at $P$. Then the following conditions are equivalent:

$R$ satisfies the right Ore condition with respect to $\mathscr{C}(P)$ and, for each right ideal $E$ of $R_{P}$, there exists a natural number $n$ 
such that $E \cap M^{n} \subseteq E M$.

(**) $R_{P}$ is a classical quasi-local ring with maximal ideal $M$.

(***) $M$ is a two-sided ideal of $R_{P}, R_{P} / M$ is a simple Artinian ring, and on any finitely generated right $R_{P}$-module the $I_{P}$-adic and M-adic topologies coincide.

(****) $M$ is a two-sided ideal of $R_{P}, R_{P} / M$ is simple Artinian, and for each finitely generated right ideal $E$ of $R_{P}$ there exists a natural number $n$ such that $E \cap M^{n} \cong E M$.

Proof. We shall show that $\left({ }^{*}\right) \Rightarrow\left({ }^{* *}\right) \Rightarrow\left({ }^{* * *}\right) \Rightarrow\left({ }^{* * * *}\right) \Rightarrow\left({ }^{*}\right)$.

Assume $\left(^{*}\right)$. In view of [7], Theorem 5.6, $\left({ }^{* *}\right)$ will follow if we show that every right ideal $F$ of $R_{P}$ is closed in the $M$-adic topology. Now its closure is given by $E=\bigcap_{n=1}^{\infty}\left(F+M^{n}\right)$. Pick $n$ so that $E \cap$ $M^{n} \cong E M$, then

$$
E \subseteq\left(F+M^{n}\right) \cap E=F+\left(M^{n} \cap E\right) \subseteq F+E M .
$$

Take any $e \in E$, then $e=f+\sum_{i=1}^{k} e_{i} m_{i}$, where $f \in F, e_{i} \in E$, and $m_{i} \in$ $M$. Then $[e]=\sum_{i=1}^{k}\left[e_{i}\right] m_{i}$, modulo $F$, hence $E / F \subseteq(E / F) M$.

It was pointed out in the discussion preceding [7], Theorem 5.6, that $R_{P}$ is right Noetherian. Thus $E$ and $E / F$ are finitely generated $R_{P}$-modules. We may therefore invoke Nakayama's Lemma and deduce that $E / F=0$. Thus $F=E$, and so $\left({ }^{* *}\right)$ holds.

Assume $\left(^{* *}\right)$. By Lemma $4, M$ is the Jacobson radical of $R_{P}$. By [7], Theorem 5.6, $R$ satisfies the right Ore condition with respect to $\mathscr{C}(P)$. Let $G$ be any finitely generated right $R_{P}$-module. Then, by Proposition 1 , the $I_{P}$-adic topology on $G$ contains the $M$-adic topology. By Proposition 2 and [4] Theorem 5.3, the converse is true. Thus $(* * *)$ holds.

Assume $\left({ }^{* *}\right)$. Suppse $E$ is any finitely generated right ideal of $R_{P}$. Then $E M$ is an open subset of $E$ in the $M$-adic, hence in the $I_{P}$-adic topology. Now the $I_{P}$-adic topology on any module induces the $I_{P}$-adic topology on any submodule. Therefore, $E M=E \cap V$, where $V$ is an open subset of $R_{P}$ in the $I_{P}$-adic topology. Since $R_{P}$ is a finitely generated $R_{P}$-module, $V$ is an open set in the $M$-adic topology, hence $M^{n} \subseteq V$ for some $n$, and so $E \cap M^{n} \subseteq E \cap V=E M$. Thus (****) holds.

Assume $\left({ }^{* * *}\right)$. It remains to prove the right Ore condition. Given $a \in R$ and $c \in \mathscr{C}(P)$, we see from Proposition 3 that, for each positive integer $n$, there exist $a_{n} \in R$ and $c_{n} \in \mathscr{C}(P)$ such that $h\left(a c_{n}-c a_{n}\right)=$ $h\left(u_{n}\right) \in M^{n} \cap h(R)$.

Let $F$ be the right ideal generated by the $u_{n}$, then $F=u_{1} R+$ $\cdots+u_{k} R$, since $R$ is right Noetherian. Taking $E=F R_{P}$ in the above, we see that $F R_{P} \cap M^{n} \subseteq F M$, for some $n$. Hence $h\left(u_{n}\right)=u_{1} m_{1}+\cdots+$ $u_{k} m_{k}$, where the $m_{i} \in M$. 
Pick $d \in \mathscr{C}(P)$ so that all $m_{i} d \in h(R)$, then $m_{i} d \in M \cap h(R)=h(P)$, and we may write $m_{i} d=h\left(p_{i}\right)$, where $p_{i} \in P$.

Put $c^{\prime}=c_{n} d-\sum_{i=1}^{k} c_{i} p_{i}$ and $a^{\prime}=a_{n} d-\sum_{i=1}^{k} a_{i} p_{i}$, then an easy calculation shows that $h\left(a c^{\prime}\right)=h\left(c a^{\prime}\right)$. Moreover $C^{\prime} \in \mathscr{C}(P)$, since $c_{n} d \epsilon$ $\mathscr{C}(P)$ and $\sum c_{i} p_{i} \in P$. Since $a c^{\prime}-c a^{\prime} \in \operatorname{Ker} h$, we can find $d^{\prime} \in \mathscr{C}(P)$ so that $\left(a c^{\prime}-c a^{\prime}\right)^{\prime}=0$, hence $a\left(c^{\prime} d^{\prime}\right)=c\left(a^{\prime} d^{\prime}\right)$. This establishes the right Ore condition for $R$, and our proof is complete.

THEOREM 6. Let $P$ be a two-sided prime ideal of the right Noetherian ring $R$ such that $R_{P}$ is a classical quasi-local ring with maximal ideal $M=P R_{P}$. Then

(a) the M-adic completion $\hat{R}_{P}$ of $R_{P}$ is the bicommutator of the $P$-torsionfree indecomposable injective $R$-module $I_{P}$ with associated prime $P$,

(b) $\hat{R}_{P}$ is a ring of $n \times n$ matrices over a complete local ring $\hat{D}$ whose Jacobson radical $J$ is finitely generated.

Proof. (a) By Theorem 5, $R$ satisfies the right Ore condition with respect to $\mathscr{C}(P)$. By [7], Theorem 5.6, every torsionfree $R$-module is $P$-divisible. In view of [6], Proposition $2, R_{P}$ is therefore a dense subring of the bicommutator $S$ of $I_{P}$ with respect to the finite topology, as the $P$-torsion theory coincides with that determined by $I_{P}$, by [7], Corollary 3.10. By [6], Corollary 1, the finite topology coincides with the $I_{P}$-adic topology on $R_{P}$, and $S$ is the completion of $R_{P}$. By Theorem 5 , the $I_{P}$-adic topology on $R_{P}$ coincides with the $M$-adic one. Therefore $S$ is the $M$-adic completion of $R_{P}$.

(b) follows immediately from (a) and [9], Corollary 2.7.

REMARK 7. By [9], Remark 3, there exists a right Noetherian ring $R$ with a two-sided prime ideal $P$ such that $R$ satisfies the right Ore condition with respect to $\mathscr{C}(P)$, even though $R_{P}$ is not a classical quasi-local ring with maximal ideal $M$. In that example $R_{P}$ is not Hausdorff with respect to the $M$-adic topology, hence the bicommutator of $I_{P}$ is not the $M$-adic completion of $R_{P}$.

Thus the right Ore condition does not imply the second part of (*) in Theorem 5. Conversely, Example 5.9 of [7] shows that the second part of $\left(^{*}\right)$ does not imply the right Ore condition.

We conclude by giving some classes of examples satisfying the condition of Theorem 5. But first we note that, in view of Theorem 3.3 of [9], each of these is also equivalent to the following, which involves only the ring $R$ itself:

$(+)$ For every right ideal $F$ of $R$ there exists a positive integer $n$ such that $F \cap P^{(n)} \leqq \mathrm{cl}_{P}(F P)$, where $P^{(n)}$ is the $n$th right symbolic power of $P$. 
For notation see [9].

COROLlaRy 8. Let $R$ be the enveloping algebra of a finitely generated nilpotent Lie algebra, and assume that $P$ is a nonzero prime ideal of $R$. Then the conclusions (a) and (b) of Theorem 6 hold.

Proof. In Theorem 2.6 of [9], it is shown that, if $R$ is right and left Noetherian, $P^{(n)}$ coincides with the symbolic $n$th power defined by Goldie in [4]. To deduce (+), we therefore refer to [2], namely to Theorem 6, Corollary 7 and Remark I.

COROLlaRY 9. Let $R=A G$ be the group ring of a finite group $G$ over a right Noetherian prime ring $A$ of characteristic zero, and let $P$ be the augmentation ideal of $R$. Then the conclusions (a) and (b) of Theorem 6 hold.

Proof. Condition (+) holds by Corollary 3.7 of [9].

Actually, in this example $R_{P}$ is the classical ring of right quotients of $R$, and $M=P R_{P}=0$, because $P$ is the $P$-torsion ideal of $R$.

CoROLlaRY 10. Let $R$ be a right and left Noetherian hereditary prime ring, and assume that $P$ is not idempotent. Then the conclusions (a) and (b) of Theorem 6 hold. Furthermore, $\hat{D}$ is a complete discrete rank one valuation ring.

Proof. By [11], $R / P$ is a simple Artinian ring. It is known that $P$ is an invertible ideal. By Lemma 1.1 of [3], it then follows that $P$ has the Artin-Rees property. Now, by Corollary 2.8 of [9], $P^{n}=$ $P^{(n)}$, hence condition $(+)$ holds.

It remains to show that $\hat{D}$ is a rank one valuation ring. By the remark preceding Theorem 5.6 in [7], $R_{P}$ is hereditary Noetherian and quasi-local. As is well-known, this implies that $\hat{R}_{P}$ is hereditary Noetherian. By Morita equivalence, $\hat{D}$ is hereditary Noetherian. But it is local, hence a discrete rank one valuation ring.

For the sake of completeness, we shall show that $P$ is invertible. Let $Q$ be the maximal ring of right and left quotients of $R$ and put $R \cdot \cdot P=\{q \in Q \mid q P \subseteq R\}$. It is known [3] that $R \cong P(R . \cdot P)$ provided $P$ is finitely generated and projective as a right $R$-module and "dense" in a technical sense, which means that $P$ has zero left annihilator in $R$ when $P$ is a two-sided ideal. Since $R$ is right Noetherian, right hereditary, and prime, $P$ satisfies all three conditions.

Now $P \subseteq(R \cdot \cdot P) P \subseteq R$, and $P$ is maximal. Therefore $(R \cdot \cdot P) P=$ $P$ or $R$. Suppose the former, then $P \subseteq P(R . \cdot P) P=P^{2}$, which would 
lead to the contradiction that $P$ is idempotent. Therefore, $(R . \cdot P) P=R$. Finally, consider $P \cdot . R=\{q \in Q \mid P q \subseteq R\}$. Then

$$
P \cdot R=(R \cdot \cdot P) P(P \cdot . R) \subseteq R \cdot P \text {. }
$$

By symmetry we obtain $P(P \cdot R)=R$ and $R \cdot \cdot P \subseteq P \cdot . R$, and so $P$ is invertible in $Q$.

For the sake of completeness, we shall also include the argument of [1] to show that $P$ has the Artin-Rees property. Let $E$ be any right ideal of $R$ and put $E_{k}=\left(E \cap P^{k}\right) P^{-k}$. Since $R$ is right Noetherian, there exists a positive integer $k$ such that $E_{k} \subseteq E_{1}+\cdots+E_{k-1}$. Then $E \cap P^{k}=E_{k} P^{k} \subseteq \sum_{i<k}\left(E \cap P^{i}\right) P^{k-i} \sqsubseteq E P$, and this is the Artin-Rees property.

\section{REFERENCES}

1. A. W. Chatters and S. M. Ginn, Localization in hereditary rings, J. Algebra, 22 (1972), 82-88.

2. J. C. Connell, Localization in enveloping rings, J. London Math. Soc., 43 (1968), 421-428.

3. G. D. Findlay, Multiplicative ideal theory and rings of quotients, I, Proc. Roy. Soc. Edinburgh, 68 (1968), 30-53.

4. A. W. Goldie, Localization in non-commutative Noetherian rings, J. Algebra, 5 (1967), 89-105.

5. J. Kuzmanovich, Completions of Dedekind prime rings and second endomorphism rings, Pacific J. Math., 36 (1971), 721-729.

6. J. Lambek, Bicommutators of nice injectives, J. Algebra, 21 (1972), 60-73.

7. J. Lambek and G. Michler, The torsion theory at a prime ideal of a right Noetherian ring, J. Algebra, 25 (1973), 364-389.

8. E. Matlis, Injective modules over Noetherian rings, Pacific J. Math., 8 (1958), 511528.

9. G. Michler, Right symbolic powers and classical localization in right Noetherian rings, Math. Z., 127 (1972), 57-69.

10. L. Small, Orders in Artinian rings II, J. Algebra, 9 (1968), 266-273.

11. D. B. Webber, Ideals and modules of simple Noetherian hereditary rings, J. Algebra, 16 (1970), 239-242.

Received June 7, 1972.

McGill University

AND

UNIVERSITÄt GIESSEN 


\section{PACIFIC JOURNAL OF MATHEMATICS}

\section{EDITORS}

RICHARD ARENS (Managing Editor)

University of California

Los Angeles, California 90024

R. A. Beaumont

University of Washington

Seattle, Washington 98105
J. DUGUNDJI*

Department of Mathematics

University of Southern California

Los Angeles, California 90007

D. Gilbarg and J. Milgram

Stanford University

Stanford, California 94305

\section{ASSOCIATE EDITORS}
E. F. BECKENBACH
B. H. NeumanN
F. WOLF
K. YosHIDA

\section{SUPPORTING INSTITUTIONS}

UNIVERSITY OF BRITISH COLUMBIA
CALIFORNIA INSTITUTE OF TECHNOLOGY
UNIVERSITY OF CALIFORNIA
MONTANA STATE UNIVERSITY
UNIVERSITY OF NEVADA
NEW MEXICO STATE UNIVERSITY
OREGON STATE UNIVERSITY
UNIVERSITY OF OREGON
OSAKA UNIVERSITY

UNIVERSITY OF BRITISH COLUMBIA

UNIVERSITY OF CALIFORNIA

MONTANA STATE UNIVERSITY

UNIVERSITY OF NEVADA

OREGON STATE UNIVERSITY

OSAKA UNIVERSITY
UNIVERSITY OF SOUTHERN CALIFORNIA

STANFORD UNIVERSITY

UNIVERSITY OF TOKYO

UNIVERSITY OF UTAH

WASHINGTON STATE UNIVERSITY

UNIVERSITY OF WASHINGTON

\section{AMERICAN MATHEMATICAL SOCIETY} NAVAL WEAPONS CENTER

* C. R. DePrima California Institute of Technology, Pasadena, CA 91109, will replace J. Dugundji until August 1974. 


\section{Pacific Journal of Mathematics}

\section{Vol. 48, No. $1 \quad$ March, 1973}

Jan Aarts and David John Lutzer, Pseudo-completeness and the product of Baire

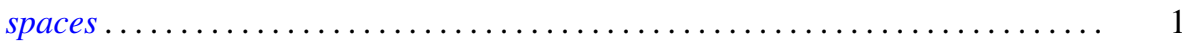

Gordon Owen Berg, Metric characterizations of Euclidean spaces ............ 11

Ajit Kaur Chilana, The space of bounded sequences with the mixed topology ..... . 29

Philip Throop Church and James Timourian, Differentiable open maps of

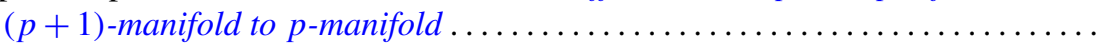

P. D. T. A. Elliott, On additive functions whose limiting distributions possess a finite

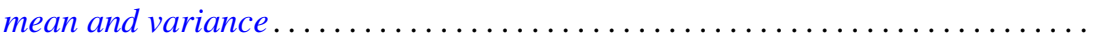

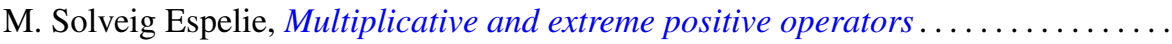

Jacques A. Ferland, Domains of negativity and application to generalized convexity

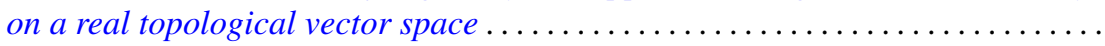

Michael Benton Freeman and Reese Harvey, A compact set that is locally holomorphically convex but not holomorphically convex ...............

Roe William Goodman, Positive-definite distributions and intertwining

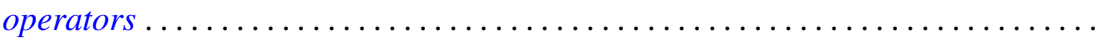

Elliot Charles Gootman, The type of some $C^{*}$ and $W^{*}$-algebras associated with

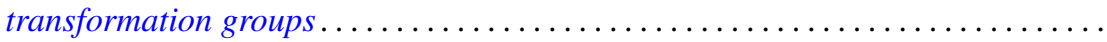

David Charles Haddad, Angular limits of locally finitely valent holomorphic

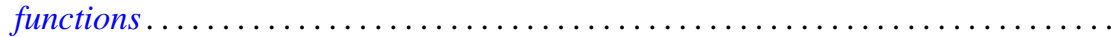

William Buhmann Johnson, On quasi-complements .

William M. Kantor, On 2-transitive collineation groups of finite projective spaces...

Joachim Lambek and Gerhard O. Michler, Completions and classical localizations of right Noetherian rings

Kenneth Lamar Lange, Borel sets of probability measures ......

David Lowell Lovelady, Product integrals for an ordinary differential equation in a Banach space

Jorge Martinez, A hom-functor for lattice-ordered groups .........

W. K. Mason, Weakly almost periodic homeomorphisms of the two sphere ....

Anthony G. Mucci, Limits for martingale-like sequences .......

Eugene Michael Norris, Relationally induced semigroups ...

Arthur E. Olson, A comparison of c-density and $k$-density ......

Donald Steven Passman, On the semisimplicity of group rings of linear groups.

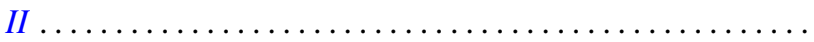

Charles Radin, Ergodicity in von Neumann algebras .

P. Rosenthal, On the singularities of the function generated by the Bergman operator of the second kind.

Arthur Argyle Sagle and J. R. Schumi, Multiplications on homogeneous spaces,

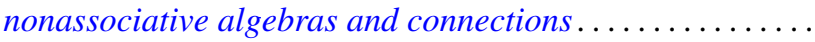

Leo Sario and Cecilia Wang, Existence of Dirichlet finite biharmonic functions on

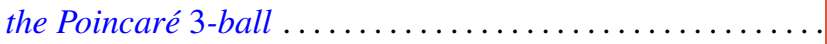

Ramachandran Subramanian, On a generalization of martingales due to Blake ..

Bui An Ton, On strongly nonlinear elliptic variational inequalities.

Seth Warner, A topological characterization of complete, discretely valued

fields. 